\title{
HIGH DENSITY POLYETHYLENE/CARBON NANOTUBE NANOCOMPOSITE FOAMS: ELECTRICAL CONDUCTIVITY AND PERCOLATION THRESHOLD
}

\author{
Linh T. Hoang \\ Department of Physics \& Astronomy \\ York University \\ Toronto, ON, Canada M3J 1P3 \\ linhhlp@yorku.ca
}

\author{
Mohammadmehdi Aghelinejad, Siu N. Leung, and \\ Zheng H. Zhu \\ Department of Mechanical Engineering \\ Lassonde School of Engineering, York University \\ Toronto, ON, Canada M3J 1P3 \\ sunny.leung@lassonde.yorku.ca
}

\begin{abstract}
Electrically conductive polymer/carbon nanotube nanocomposites have drawn attention for a wide range of applications because of their light weight, good chemical resistance, ease of processing, and tunable multifunctional properties. High density polyethylene (HDPE)/multiwalled carbon nanotube (MWCNT) nanocomposites and their foams were fabricated, and their electrical properties were explored in this research. Parametric studies were conducted to investigate the effects of initial MWCNT loading and processing conditions on the foaming behaviors of HDPE/MWCNT nanocomposites. Experimental results revealed that both the initial MWCNT loading and foam morphology were governing factors to tune the electrical conductivity and the percolation threshold of HDPE/MWCNT nanocomposites.
\end{abstract}

Keywords-electrical conductivity; foam morphology; multiwalled carbon nanotubes; percolation threshold; polymer nanocomposites

\section{INTRODUCTION}

Polymer/carbon nanotube (CNT) nanocomposites have drawn extensive interests from researchers and industries because of their low density, superior chemical resistance, good manufacturability, and tunable multifunctional properties. As a result, this versatile material system has found a wide spectrum of applications including electromagnetic interference shielding [1], energy harvesting [2], energy storage [3], sensors [4-6], and thermal management [7]. Recent studies have suggested foaming polymer composites and nanocomposites as a promising fabrication strategy to tailor their micro-andnanostructures. Through biaxial stress fields induced by foam expansion, it is possible to align both one-dimensional (1D) and two-dimensional (2D) multifunctional fillers and thereby promote the material system's mechanical [8], electrical [9], and thermal [10] properties.

The electrical conductivity of a polymer/CNT nanocomposite can be drastically improved once its CNT loading has increased above a critical level, denoted as percolation threshold $\left(p_{c}\right)$. While high CNT loading promotes nanocomposites' electrical conductivity, it is detrimental to the weight, processability, and mechanical properties of
polymer/CNT nanocomposites. In this context, extensive studies were conducted to develop strategies for promoting polymer/CNT nanocomposite's electrical conductivity with lower CNT loading. These includes the utilization of hybrid conductive fillers with different types, sizes, and/or shapes as well as using immiscible polymer blends. Park et al. [11] investigated the effect of hybrid fillers on the electrical conductivity of the polypropylene (PP) composite. In their investigation, the inclusion of both MWCNT and carbon fiber in the PP matrix led to better conductivity than the case filled with only carbon fiber. In another study [12], acrylonitrile butadiene styrene (ABS) was melt-blended in polycarbonate (PC)/ MWCNT nanocomposite. Due to the partial solubility of ABS in PC, the selective dispersion of the MWCNTs in the ABS phase significantly promoted the electrical conductivity while reducing $p_{c}$.

This paper reports the application of supercritical carbon dioxide $\left(\mathrm{ScCO}_{2}\right)$ foaming to tailor the nanostructure of high density polyethylene (HDPE)/multiwalled carbon nanotube (MWCNT) nanocomposites. Parametric studies were conducted to elucidate the processing-structure-property relationships of HDPE/MWCNT nanocomposites and their foams. The effects of initial MWCNT loading and foaming temperature on the foam morphology and MWCNT networking of HDPE/MWCNT nanocomposites were studied. Furthermore, the interrelations among HDPE/MWCNT nanocomposite's foam morphology, MWCNT localization and networking, as well as the material system's electrical conductivity and percolation threshold were systematically studied. This work provides important guidelines to design and fabricate polymer/carbon nanotube nanocomposites with enhanced electrical conductivity and suppressed percolation threshold.

\section{EXPERIMENTAL}

\section{A. Materials}

Commercially available HDPE/MWCNT nanocomposite masterbatch (Nanocyl, Plasticyl HDPE1501), loaded with 15 wt\% MWCNT (Nanocyl, NC7000 ${ }^{\mathrm{TM}}$ ), and neat HDPE (NOVA Chemicals, SCLAIR ${ }^{\circledR}$ 2710) were used to prepare HDPE/MWCNT nanocomposites of different MWCNT 
loadings. The constituent MWCNT had an average length of 1.5 $\mu \mathrm{m}$ and an average diameter of $9.5 \mathrm{~nm}$. The physical foaming agent used in this study was carbon dioxide $\left(\mathrm{CO}_{2}\right)$ (Linde Gas Inc., 99.8\% purity). Tables I through III summarize the physical properties of HDPE/MWCNT masterbatch, HDPE, and MWCNT, respectively. All materials were used as received without any modification.

\section{B. Sample Preparation}

Calculated amounts of HDPE/MWCNT nanocomposite masterbatch were weighed and the MWCNT content were reduced to various levels by adding different amounts of neat HDPE. The dilution was done by melt compounding the mixtures using a micro-compounder (HAAKETM MiniCTW Micro-Conical Twin Screw Compounder) in the circulating mode for 5 minutes at $215^{\circ} \mathrm{C}$, with the screw speed set at $75 \mathrm{rpm}$. The extrudates were pelletized and compression molded into a circular disc sample of $115 \mathrm{~mm}$ in diameter and $0.50 \mathrm{~mm}$ in thickness using a compression molding machine (Craver Press,

TABLE I. PHYSICAL PROPERTIES OF HDPE/MWCNT NANOCOMPOSITE MASTERBATCH

\begin{tabular}{|l|c|c|}
\hline \multicolumn{1}{|c|}{ Property } & Value & Unit \\
\hline MWCNT Loading & 15 & $\mathrm{wt} \%$ \\
\hline Density & 977 & $\mathrm{~kg} / \mathrm{m}^{3}$ \\
\hline Melting Temperature & 135 & ${ }^{\circ} \mathrm{C}$ \\
\hline Resistivity & $1.0(\max )$ & $\mathrm{k} \Omega$ \\
\hline
\end{tabular}

TABLE II. PHySICAL PROPERTIES OF NEAT HDPE

\begin{tabular}{|l|c|c|}
\hline \multicolumn{1}{|c|}{ Property } & Value & Unit \\
\hline Density & 951 & $\mathrm{~kg} / \mathrm{m}^{3}$ \\
\hline Vicat Softening Temperature & 125 & ${ }^{\circ} \mathrm{C}$ \\
\hline Resistivity & $1.0(\max )$ & $\mathrm{k} \Omega$ \\
\hline
\end{tabular}

TABLE III. PHYSICAL PROPERTIES OF MWCNT

\begin{tabular}{|l|c|c|}
\hline \multicolumn{1}{|c|}{ Property } & Value & Unit \\
\hline Average Diameter & 9.5 & $\mathrm{~nm}$ \\
\hline Average Length & 1.5 & $\mu \mathrm{m}$ \\
\hline Carbon Purity & 90 & $\%$ \\
\hline Surface Area & $250-300$ & $\mathrm{~m}^{2} / \mathrm{g}$ \\
\hline Volume Resistivity & $10^{-4}$ & $\Omega \cdot \mathrm{cm}$ \\
\hline
\end{tabular}

TABLE IV. PARAMETERS STUdIED IN PhySCIAL FOAMING EXPERIMENTS

\begin{tabular}{|l|c|c|}
\hline \multicolumn{1}{|c|}{ Parameter } & Value & Unit \\
\hline MWCNT Loading & $1,2,3,5,7$, and 10 & wt $\%$ \\
\hline$P_{\text {sat }}$ & 1,200 (fixed) & $\mathrm{psi}$ \\
\hline$T_{\text {sat }}$ & $123-135$ & ${ }^{\circ} \mathrm{C}$ \\
\hline Saturation Time & 30 (fixed) & $\min$ \\
\hline
\end{tabular}

$4386 \mathrm{CH})$. The compression molding procedure is summarized below:

STEP 1. Weighed amount of HDPE/MWCNT nanocomposite pellets were loaded into a circular disc mold of $115 \mathrm{~mm}$ in diameter and $0.50 \mathrm{~mm}$ in thickness.

STEP 2. The compression molding machine was equilibrated at $155^{\circ} \mathrm{C}$.

STEP 3. The heat platens of the compression molding machine were brought into contact with the mold, which loaded with the nanocomposite pellets, for 5 minutes to ensure the complete melting of the nanocomposite pellets.

STEP 4. The molding pressure was ramped up to 4,000 psi and held at this level for 6 minutes.

STEP 5. The disc samples, which were still inside the mold, were transferred to a cooling module with flowing water channels to solidify the disc samples.

STEP 6. The samples were cut into rectangular specimens with dimensions of $12 \mathrm{~mm} \times 10 \mathrm{~mm} \times 0.50 \mathrm{~mm}$. The specimens would then be characterized using an electrical conductivity analyzer or be foamed by $\mathrm{ScCO}_{2}$.

HDPE/MWCNT nanocomposite foams were fabricated by a batch foaming chamber using $\mathrm{ScCO}_{2}$ as the physical foaming agent. In the physical foaming process, nanocomposite specimens were saturated with $\mathrm{ScCO}_{2}$ for 30 minutes at preset combinations of saturation pressure $\left(P_{\text {sat }}\right)$ and saturation temperature $\left(T_{\text {sat }}\right)$. Subsequently, an outlet valve of the batch foaming chamber was opened to rapidly depressurize it. This led to thermodynamic instability in the $\mathrm{HDPE} / \mathrm{MWCNT} / \mathrm{ScCO}_{2}$ system, and thereby induced cell nucleation and subsequent cell growth in the HDPE matrices. Table IV summarizes the key parameters being studied in the physical foaming experiments.

\section{Sample Characterization}

The apparent density of MWCNT/HDPE nanocomposite foams, were determined in accordance with ASTM D792. After measuring their masses in air and in water, the apparent density of a foam sample $\left(\rho_{f}\right)$ and the volume expansion ratio $(\phi)$ can be determined by Equations (1) and (2), respectively.

$$
\rho_{f}=\frac{m_{\text {air }} \rho_{\text {water }}}{m_{\text {air }}-m_{\text {water }}}
$$

where $m_{\text {air }}$ and $m_{\text {water }}$ are the masses of samples measured in air and in water, respectively; and $\rho_{\text {water }}$ is the density of water.

$$
\phi=\frac{\rho_{s}}{\rho_{f}}
$$

where $\rho_{s}$ is the density of the solid HDPE/MWCNT nanocomposite sample.

The phase and foam morphologies of the solid and foamed HDPE/MWCNT nanocomposite samples were characterized by scanning electron microscopy (SEM) (FEI Company Quanta 3D FEG). The cross-sections of all samples were exposed by cryo-fracturing the samples under liquid nitrogen. The fractured 
surfaces were then sputter-coated with gold (Denton Vacuum, Desk V Sputter Coater). The cell size and cell population density of selected foam samples were obtained by analyzing the SEM micrographs of the foams using Image $\mathbf{J}$ (National Institute of Health). The cell population density $\left(N_{0}\right)$ with respect to the unfoamed volume was determined by Equation (3).

$$
N_{0}=\phi \times\left[\frac{n M^{2}}{A}\right]^{\frac{3}{2}}
$$

where $n$ is the number of cells in the SEM micrograph; $M$ is the magnification factor; and $A$ is the area of the micrograph.

The electrical conductivity of solid HDPE/MWCNT nanocomposite samples were measured by the two-wire technique using a multifunctional source meter (Keithley, 2450 Source Meter). The two-wire technique was performed in accordance with the ASTM D257-07 standard. By printing conductive silver-epoxy paste as electrodes on both ends of a sample, the voltage difference was introduced through the sample and the flowing current was recorded to calculate the electrical conductivity of the nanocomposite sample. Depending on the volume expansion ratios, the thicknesses of foamed nanocomposite samples varied from 0.50 to $2.5 \mathrm{~mm}$. Fig. 1 illustrates the schematic of a nanocomposite sample prepared for the two-wire technique.

\section{RESULTS AND DISCUSSION}

Parametric studies were conducted to elucidate the processing-structure-property relationships of HDPE/MWCNT nanocomposite foams. On the one hand, it was expected that the presence of MWCNT would influence the $\mathrm{ScCO}_{2}$ foaming of HDPE. On the other hand, the biaxial stress fields induced by foam expansion would affect the alignment and localization of MWCNT in the HDPE matrix.

\section{A. Micro-and-Nanostructures of HDPE/MWCNT \\ Nanocomposites and Their Foams}

Fig. 2 (a) and (b) illustrate representative SEM micrographs of HDPE/MWCNT nanocomposites loaded with $3 \mathrm{wt} \%$ and 10 wt\% MWCNT, respectively. While increasing MWCNT loadings would promote the interconnectivity of MWCNT in HDPE matrices, it can be observed that continuous networks of MWCNT were omnipresent in the HDPE matrix even at $3 \mathrm{wt} \%$ MWCNT loading.

Fig. 3 (a) through (f) show the SEM micrographs of HDPE/MWCNT loaded with $3 \mathrm{wt} \%$ MWCNT before subjected to $\mathrm{ScCO}_{2}$ foaming at $P_{\text {sat }}$ of $1,200 \mathrm{psi}$ and different $T_{\text {sat }}$. Experimental observation revealed that foam morphologies of HDPE/MWCNT nanocomposite foams had strong dependence on $T_{\text {sat }}$. At $123^{\circ} \mathrm{C}$, the HDPE matrix, reinforced by MWCNT, was too stiff to achieve observable foam expansion. As $T_{\text {sat }}$ gradually increased, the foam morphologies evolved from having a high cell density with fine cells to a reduced cell density with larger cells. The foam structure was finally deteriorated when $T_{\text {sat }}$ was increased to $135^{\circ} \mathrm{C}$. In other words, within the processing window, varying $T_{\text {sat }}$ would be an effective approach to tune the cell morphologies and thereby the MWCNT network of HDPE/MWCNT nanocomposite foams.

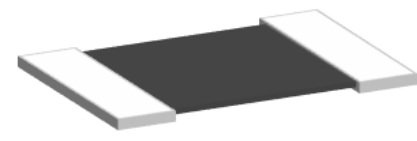

Figure 1. A Schematic of a Nanocomposite Sample Prepared for Electrical Conductivity Measurement by Two-Wire Technique

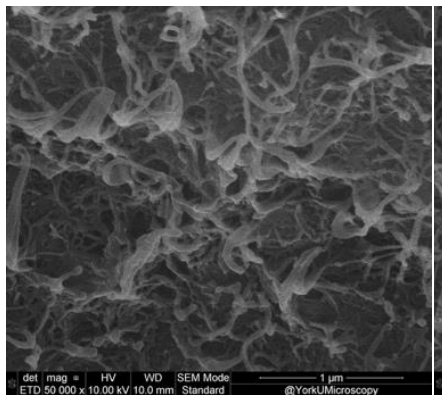

(a)

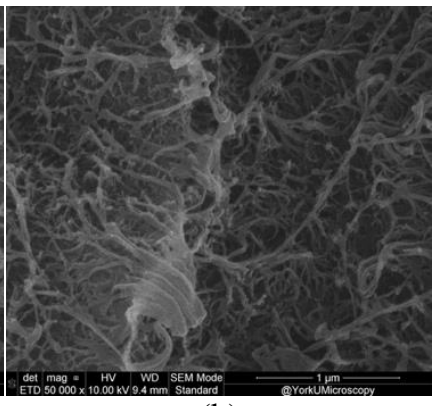

(b)
Figure 2. SEM Micrographs of HDPE/MWCNT Nanocomposites Loaded with (a) $3 \mathrm{wt} \%$ and (b) $10 \mathrm{wt} \%$ MWCNT

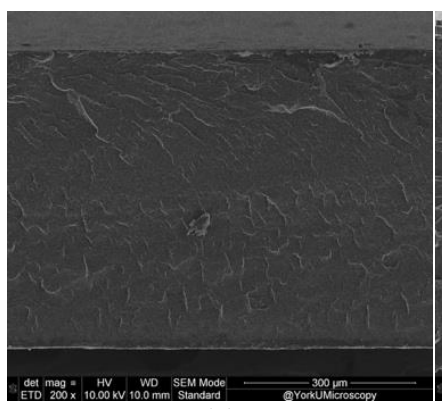

(a)

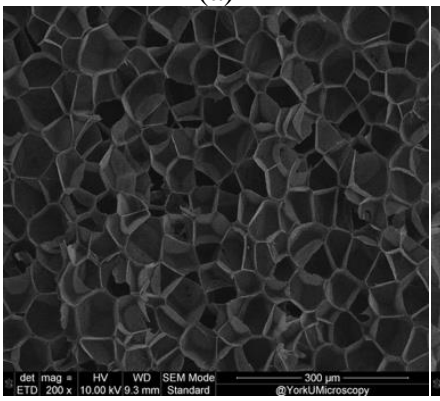

(c)

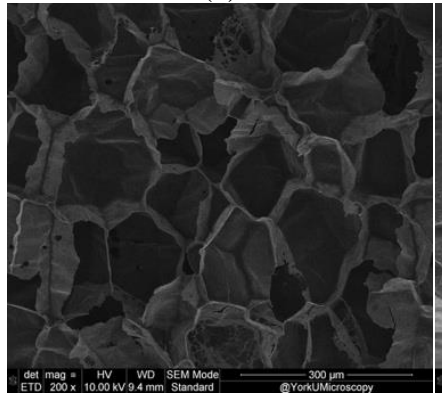

(e)

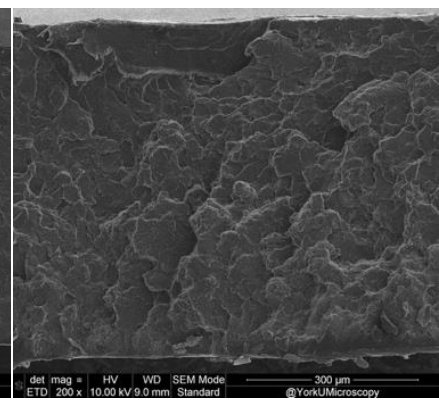

(b)

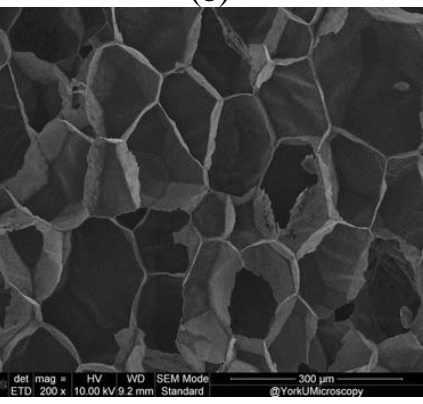

(d)

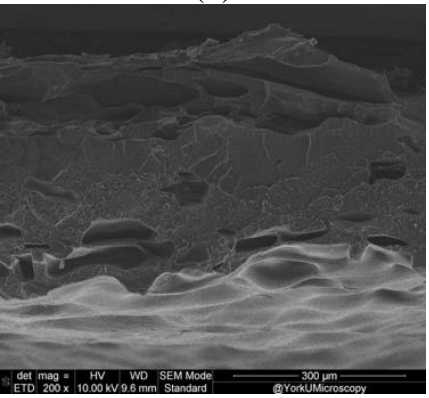

(f)
Figure 3. SEM Micrographs of HDPE/MWCNT Nanocomposite and Foams Loaded with $3 \mathrm{wt} \%$ Fabricated at $P_{\text {sat }}$ of 1,200 psi and different $T_{\text {sat }}$ : (a) Solid Nanocomposite; (b) $123^{\circ} \mathrm{C}$; (c) $125^{\circ} \mathrm{C}$; (d) $127^{\circ} \mathrm{C}$; (e) $130^{\circ} \mathrm{C}$; and (f) $135^{\circ} \mathrm{C}$ 


\section{B. Foaming Behaviours of HDPE/MWCNT Nanocomposites}

Experimental observation revealed that the optimal $T_{\text {sat }}$, to maximize the volume expansion of HDPE/MWCNT nanocomposite foams, depended on the initial loading of MWCNT. According to Fig. 4, by increasing the MWCNT content of the nanocomposites from 1 to $10 \mathrm{wt} \%$, their optimal $T_{\text {sat }}$ value also increases as the nanocomposite's melting temperature rises by increasing the MWCNT loadings. This could be attributed to the increased melt strength of the cell wall and reduced $\mathrm{CO}_{2}$ diffusivity as MWCNT loading increased.

\section{Electrical Conductivity and Percolation Thresholds of HDPE/MWCNT Nanocomposites and Their Foams}

To compare solid and foamed composites, the loadings of MWCNT were converted from weight fraction to volume fraction by considering the MWCNT density to be $1.75 \mathrm{~g} / \mathrm{cm}^{3}$ [9]. The volume fraction of MWCNT in foamed samples were calculated with respect to the total volumes of the solid part and the voids. Fig. 5 illustrates the effects of volume fractions of MWCNT on the electrical conductivity of HDPE/MWCNT nanocomposites and their foams at different initial MWCNT loadings (i.e., 3, 5, and $10 \mathrm{wt} \%$ ). Regarding of the initial MWCNT loading, the electrical conductivity of all nanocomposites increased with the final volume fraction of MWCNT. However, depending on the initial MWCNT content, nanocomposite foams loaded with the same final volume fraction of MWCNT showed significantly different electrical conductivity values. Experimental results also indicated that at low volume fraction of MWCNT, the electrical conductivity of foamed samples was remarkably higher than the solid samples. This increase was more significant for nanocomposites with higher MWCNT loading. As an example, the electrical conductivity of HDPE/MWCNT foam samples initially loaded with $10 \mathrm{wt} \%$ MWCNT was two orders of magnitude higher than the solid samples at $0.5 \mathrm{vol} \%$ (about $1 \mathrm{wt} \%$ ) and seven orders of magnitude higher at $0.3 \mathrm{vol} \%$ (about $0.5 \mathrm{wt} \%$ ).

The value of $p_{c}$ of the HDPE/MWCNT nanocomposites, which is the minimum MWCNT loading that would transform the nanocomposites from insulating to conductive, was estimated by fitting the percolation model (i.e., Equation (4)) to the experimentally obtained electrical conductivity data.

$$
\sigma=\sigma_{0}\left(p-p_{c}\right)^{t}
$$

where $\sigma$ is the electrical conductivity of the nanocomposite; $p$ is the loading of MWCNT; and $\sigma_{0}, p_{c}$ and $t$ were fitting constants and achieved by curve fitting using the root-mean-square-error method.

Fig. 6 presents the effect of foaming process on reducing $p_{c}$ of the solid HDPE/MWCNT nanocomposites. Experimental results indicated that $p_{c}$ of HDPE/MWCNT nanocomposites effectively decreased after foaming. The initial MWCNT content was a critical factor on suppressing the percolation threshold of the nanocomposite foams. By increasing the initial MWCNT loading of the nanocomposite samples from $3 \mathrm{wt} \%$ to $10 \mathrm{wt} \%$, the percolation threshold of the nanocomposite decreased from 0.55 vol\% to $0.36 \mathrm{vol} \%$.

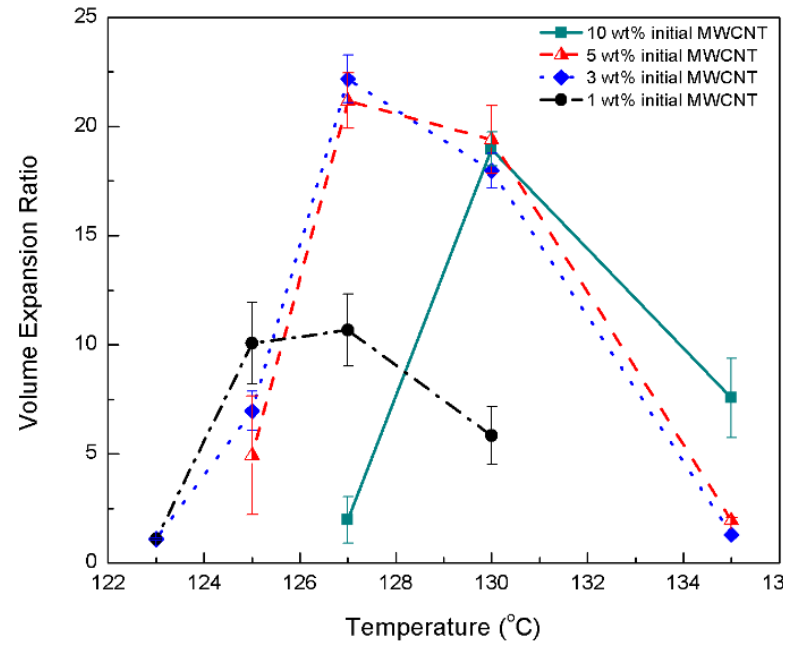

Figure 4. Effects of Tsat on Volume Expansion of HDPE/MWCNT Nanocomposite Foams with Different Initial MWCNT Loadings

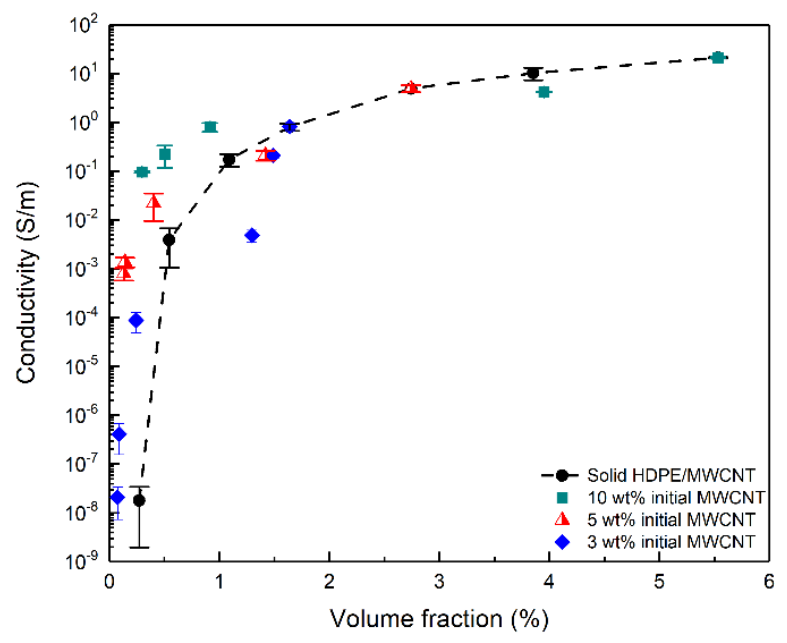

Figure 5. Effects of Volume Fractions of MWCNT on the Electrical Conductivity of HDPE/MWCNT Nanocomposites and Their Foams at different initial MWCNT loadings

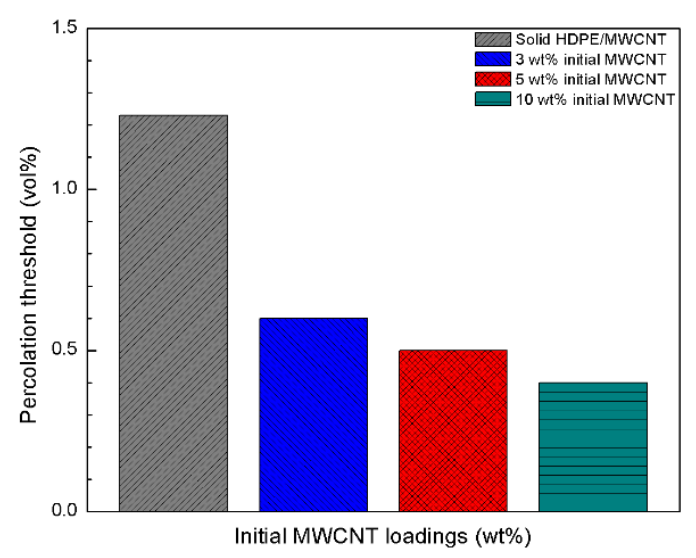

Figure 6. Percolation threshold for foams prepared with different initial MWCNT loadings vs that of solid nanocomposite 


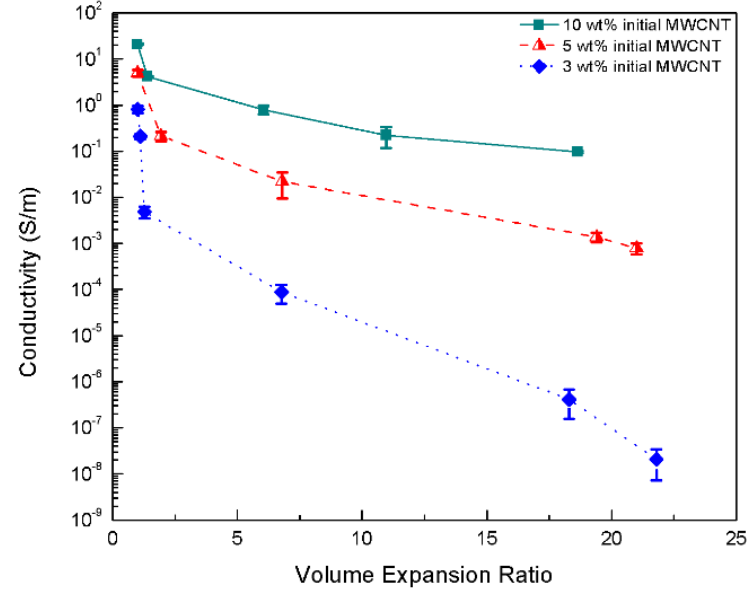

Figure 7. Effect of Volume Expansion Ratio on the Electrical Conductivity of HDPE/MWCNT Nanocomposites and Their Foams at different initial MWCNT loadings

Fig. 7 shows the dependence of electrical conductivity on the volume expansion ratio of HDPE/MWCNT nanocomposite foams. At the same initial MWCNT content, the higher expansion ratio would lead to smaller volume fractions of MWCNT, which decreased the electrical conductivity value for the foamed samples. Similar behavior was observed for all nanocomposite samples with different MWCNT initial loadings. However, it must be noted that larger volume expansion would also reduce the effective MWCNT loading in the nanocomposite foam. Overall, experimental results demonstrated that foaming was a promising strategy to increase the electrical conductivity of HDPE/MWCNT nanocomposites and to reduce the percolation threshold.

\section{CONCLUSION}

In this study, the effects of physical foaming on the electrical conductivity and the percolation threshold of HDPE/MWCNT nanocomposites were investigated. By changing the saturation temperature, one of the governing physical foaming parameters, the effects of foam morphology and volume expansion ratio on the electrical conductivity of the foamed samples were also explored in this research. The foam morphology and volume expansion ratio of nanocomposite foams were crucially dependent on both saturation temperature and initial loadings of MWCNTs in nanocomposites. The electrical conductivity of foamed samples was significantly higher than their solid counterparts, especially at low MWCNT volume fraction. The enhancement in the electrical conductivity was more pronounce for nanocomposites with higher initial MWCNT loadings. This also led to lower percolation thresholds than their solid nanocomposites.

\section{ACKNOWLEDGMENT}

The authors are grateful about the financial support by the Natural Sciences and Engineering Research Council of Canada.

\section{REFERENCES}

[1] J. M. Thomassin, C. Pagnoulle, L. Bednarz, I. Huynen, R. Jerome, et al., "Foams of polycaprolactone/MWNT nanocomposites for efficient EMI reduction," J. Mater. Chem., vol. 18, pp. 792-796, 2008.

[2] M. Aghelinejad and S. N. Leung, "Enhancement of thermoelectric conversion efficiency of polymer/carbon nanotube nanocomposites through foaming-induced microstructuring," J. Appl. Polym. Sci., vol. 134, pp. 45073, 2017

[3] C. Peng, S. Zhang, D. Jewell, and G. Z. Chen, "Carbon nanotube and conducting polymer composites for supercapacitors," Progress in Natural Science, vol. 18, pp. 777-788, 2008.

[4] S. Gong, Z. H. Zhu, and Z. Li, "Electron tunnelling and hopping effects on the temperature coefficient of resistance of carbon nanotube/polymer nanocomposites," PCCP, vol. 19, pp. 5113-5120, 2017.

[5] S. Gong and Z. H. Zhu, "On the mechanism of piezoresistivity of carbon nanotube polymer composites," Polymer, vol. 55, pp. 4136-4149, 2014.

[6] N. H. Quang, M. Van Trinh, B.-H. Lee, and J.-S. Huh, "Effect of $\mathrm{NH}_{3}$ gas on the electrical properties of single-walled carbon nanotube bundles," Sensors and Actuators B: Chemical, vol. 113, pp. 341-346, 2006.

[7] S. N. Leung, M. O. Khan, E. Chan, H. E. Naguib, F. Dawson, et al., "Synergistic effects of hybrid fillers on the development of thermally conductive polyphenylene sulfide composites," J. Appl. Polym. Sci., vol. 127, pp. 3293-3301, 2013.

[8] M. Okamoto, P. H. Nam, P. Maiti, T. Kotaka, T. Nakayama, et al., "Biaxial Flow-Induced Alignment of Silicate Layers in Polypropylene/Clay Nanocomposite Foam," Nano Lett., vol. 1, pp. 503505, 2001.

[9] A. Ameli, M. Nofar, C. B. Park, P. Pötschke, and G. Rizvi, "Polypropylene/carbon nanotube nano/microcellular structures with high dielectric permittivity, low dielectric loss, and low percolation threshold," Carbon, vol. 71, pp. 206-217, 2014.

[10] H. Ding, Y. Guo, and S. N. Leung, "Development of thermally conductive polymer matrix composites by foaming-assisted networking of micron- and submicron-scale hexagonal boron nitride," J. Appl. Polym. Sci., vol. 133, 2016.

[11] D. H. Park, Y. K. Lee, S. S. Park, C. S. Lee, S. H. Kim, et al., "Effects of hybrid fillers on the electrical conductivity and EMI shielding efficiency of polypropylene/conductive filler composites," Macromolecular Research, vol. 21, pp. 905-910, 2013.

[12] S. Maiti, N. K. Shrivastava, and B. B. Khatua, "Reduction of percolation threshold through double percolation in melt-blended polycarbonate/acrylonitrile butadiene styrene/multiwall carbon nanotubes elastomer nanocomposites," Polym. Compos., vol. 34, pp. 570-579, 2013. 